\title{
Connection between entanglement and the speed of quantum evolution
}

\author{
J. Batle, ${ }^{1}$ M. Casas, ${ }^{1}$ A. Plastino, ${ }^{2}$ and A. R. Plastino ${ }^{3}$ \\ ${ }^{1}$ Departament de Física, Universitat de les Illes Balears and IMEDEA-CSIC, 07122 Palma de Mallorca, Spain \\ ${ }^{2}$ National University La Plata-CONICET, C.C. 727, 1900 La Plata, Argentina \\ ${ }^{3}$ Department of Physics, University of Pretoria, 0002 Pretoria, South Africa
}

(Received 1 July 2005; published 29 September 2005)

\begin{abstract}
It has been recently pointed out [V. Giovannetti, S. Lloyd, and L. Maccone, Europhys. Lett. 62, 615 (2003)] that, for certain classes of states, quantum entanglement enhances the "speed" of evolution of composite quantum systems, as measured by the time a given initial state requires to evolve to an orthogonal state. We provide here a systematic study of this effect for pure states of bipartite systems of low dimensionality, considering both distinguishable (two-qubits) subsystems, and systems constituted of two indistinguishable particles.
\end{abstract}

DOI: 10.1103/PhysRevA.72.032337

PACS number(s): 03.67.Mn, 89.70.+c, 03.65.-w, 02.50.-r

\section{INTRODUCTION}

Entanglement is one of the most fundamental features of the quantum description of nature [1-5]. In recent years it has been the focus of intense research efforts [1-13]. A state of a composite quantum system is called "entangled" if it cannot be represented as a mixture of factorizable pure states. Otherwise, the state is called separable. Entanglement constitutes a physical resource that lies at the basis of important quantum information processes [3-6], such as quantum teleportation, superdense coding, and quantum computation.

Entanglement is essential in connection both with our basic understanding of quantum mechanics and with some of its most revolutionary (possible) technological applications. Consequently, it is imperative to investigate, in detail, the relationships between entanglement and other aspects of quantum theory. In particular, it is of clear interest to explore the role played by entanglement in the dynamical evolution of composite quantum systems. It was recently discovered by Giovannetti et al. [1,2] that, in certain cases, entanglement helps to "speed up" the time evolution of composite systems. The problem of the "speed" of quantum evolution has aroused considerable interest recently, because of its relevance in connection with the physical limits imposed by the basic laws of quantum mechanics on the speed of information processing and information transmission [14-16].

The aim of the present contribution is to investigate, in detail, for bipartite systems of low dimensionality, the connection between entanglement and the speed of quantum evolution. We are going to focus our attention on (i) two qubits (distinguishable) systems and (ii) bosonic or fermionic composite (bipartite) systems of lowest dimensionality.

\section{TWO ENTANGLED DISTINGUISHABLE PARTICLES}

We are going to investigate first the case of two equal but distinguishable subsystems evolving under a local Hamiltonian. Let us then consider a two-qubits system whose evolution is governed by a (local) Hamiltonian

$$
H=H_{A} \otimes I_{B}+I_{A} \otimes H_{B},
$$

where $H_{A, B}$ have eigenstates $|0\rangle$ and $|1\rangle$ with eigenvalues 0 and $\epsilon$, respectively. That is, the eigenstates of $H$ are $|00\rangle$, $|01\rangle,|10\rangle$, and $|11\rangle$, with eigenvalues respectively equal to 0 , $\epsilon$ (twofold degenerate), and $2 \epsilon$. For pure states $|\Psi\rangle$ of our composite system, the natural measure of entanglement is the usual reduced von Neumann entropy $S\left[\rho_{A, B}\right]=-\operatorname{Tr}_{A, B}\left(\rho_{A, B} \ln \rho_{A, B}\right)$ (of either particle $A$ or particle $B$ ) where $\left.\rho_{A, B}=\operatorname{Tr}_{B, A}(|\Psi\rangle\rangle \Psi \mid\right)$. It is convenient for our present purposes to use, instead of $S\left(\rho_{A, B}\right)$ itself, the closely related concurrence $C$, given by

$$
C^{2}=4 \operatorname{det} \rho_{A, B} .
$$

Both the entanglement entropy $S\left[\rho_{A, B}\right]$ and the concurrence $C$ are preserved under the time evolution determined by the local Hamiltonian (1). Given an initial state

$$
|\Psi(t=0)\rangle=c_{0}|00\rangle+c_{1}|01\rangle+c_{2}|10\rangle+c_{3}|11\rangle,
$$

its concurrence is

$$
C^{2}=4\left|c_{0} c_{3}-c_{1} c_{2}\right|^{2}
$$

The overlap between the initial state (3) and the state at time $t$ is given by

$$
\langle\Psi(t) \mid \Psi(t=0)\rangle=\left|c_{0}\right|^{2}+\left(\left|c_{1}\right|^{2}+\left|c_{2}\right|^{2}\right) z+\left|c_{3}\right|^{2} z^{2},
$$

where $z \equiv \exp (i \epsilon t / \hbar) \equiv \exp (i \alpha)$, that is, $\alpha=(t \epsilon) / \hbar$.

Thus, the condition for the state at time $t$ to be orthogonal to the initial state is

$$
P(z)=\left|c_{0}\right|^{2}+\left(\left|c_{1}\right|^{2}+\left|c_{2}\right|^{2}\right) z+\left|c_{3}\right|^{2} z^{2}=0 .
$$

The above polynomial equation can be cast as

$$
\left|c_{3}\right|^{2}\left(z-z_{1}\right)\left(z-z_{2}\right)=0
$$

where $z_{1}$ and $z_{2}$ are the roots of $P(z)$. If the initial state (3) is to evolve to an orthogonal state, then the two roots of $P(z)$ have to be two (complex conjugate) numbers of modulus equal to one. That is $z_{1,2}=\exp ( \pm i \alpha)$. It that case we shall have

$$
\left|c_{0}\right|^{2}=\left|c_{3}\right|^{2}=\Gamma,
$$




$$
\left|c_{1}\right|^{2}+\left|c_{2}\right|^{2}=-2 \Gamma \cos \alpha .
$$

Appropriate normalization of the initial state also implies that the concomitant coefficients can be parameterized as

$$
\begin{gathered}
\left|c_{0}\right|^{2}=\left|c_{3}\right|^{2}=\Gamma, \\
\left|c_{1}\right|^{2}=-2 \delta \Gamma \cos \alpha, \\
\left|c_{2}\right|^{2}=-2(1-\delta) \Gamma \cos \alpha,
\end{gathered}
$$

with $\Gamma=1 / 2(1-\cos \alpha)$ and $\alpha \in[\pi / 2,3 \pi / 2], \delta \in[0,1]$. In other words, we have $\alpha=\arccos [(2 \Gamma-1) / 2 \Gamma]$.

The initial state's energy mean value and energy uncertainty are, respectively,

$$
\begin{gathered}
E=\langle H\rangle=\epsilon\left(\left|c_{1}\right|^{2}+\left|c_{2}\right|^{2}\right)+2 \epsilon\left|c_{3}\right|^{2}=\epsilon, \\
\Delta E=\sqrt{\left\langle H^{2}\right\rangle-\langle H\rangle^{2}} \\
=\left[\epsilon^{2}\left(\left|c_{1}\right|^{2}+\left|c_{2}\right|^{2}\right)+4 \epsilon^{2}\left|c_{3}\right|^{2}-\epsilon^{2}\right]^{1 / 2}=\epsilon \sqrt{2 \Gamma} .
\end{gathered}
$$

The time $\tau$ required to evolve into an orthogonal state admits the lower bound $[1,2]$,

$$
T_{\min }=\max \left(\frac{\pi \hbar}{2 E}, \frac{\pi \hbar}{2 \Delta E}\right),
$$

which, together with equations (10), lead to

$$
T_{\min }=\frac{\pi \hbar}{2 \epsilon \sqrt{2 \Gamma}} .
$$

The concurrence of the (pure) state under consideration, defined as $C^{2}=4\left|c_{0} c_{3}-c_{1} c_{2}\right|^{2}$ [see equations (2)-(4)] is

$$
C^{2}=4\left|\Gamma-e^{i \varphi} \sqrt{\delta(1-\delta)} 2 \Gamma \cos \alpha\right|^{2} .
$$

The modulus of the coefficients $c_{i}$ are completely determined by the two parameters $\alpha$ (or $\Gamma$ ) and $\delta$. The dependence of $C^{2}$ on the phases of the coefficients $c_{i}$ can be absorbed into one single phase $e^{i \varphi}$, thus incorporating a new parameter $\varphi$ into the expression (13) for $C^{2}$.

After some algebra, the expressions for the minimum and maximum values for the evolution time $\tau$ that are actually realized for states of a given concurrence $C^{2}$, read

$$
\frac{\tau}{T_{\min }(\Gamma)}=\frac{2}{\pi} \sqrt{2 \Gamma} \arccos \left(\frac{2 \Gamma-1}{2 \Gamma}\right),
$$

where the maximum evolution time for a fixed $C^{2}$ (or a fixed $C$ ) corresponds to $\Gamma=\sqrt{C^{2}} / 2$, while the minimum one to $\Gamma$ $=\left(1+\sqrt{C^{2}}\right) / 4$. The two curves in the $\left(C, \tau / T_{\text {min }}\right)$ plane corresponding, for each value of $C$, to the states with maximum and minimum $\tau / T_{\min }$ are depicted in Fig. 1. All states that eventually evolve into an orthogonal state (that is, states characterized by different $\delta$ 's and $\varphi$ 's) lie between these two curves. Some important features of the connection between entanglement and speed of evolution (for two qubits) transpire from Fig. 1. First, we see that both the maximum and the minimum times required to reach an orthogonal state are monotonously decreasing functions of the concurrence. Second, the difference between these maximum and the mini-

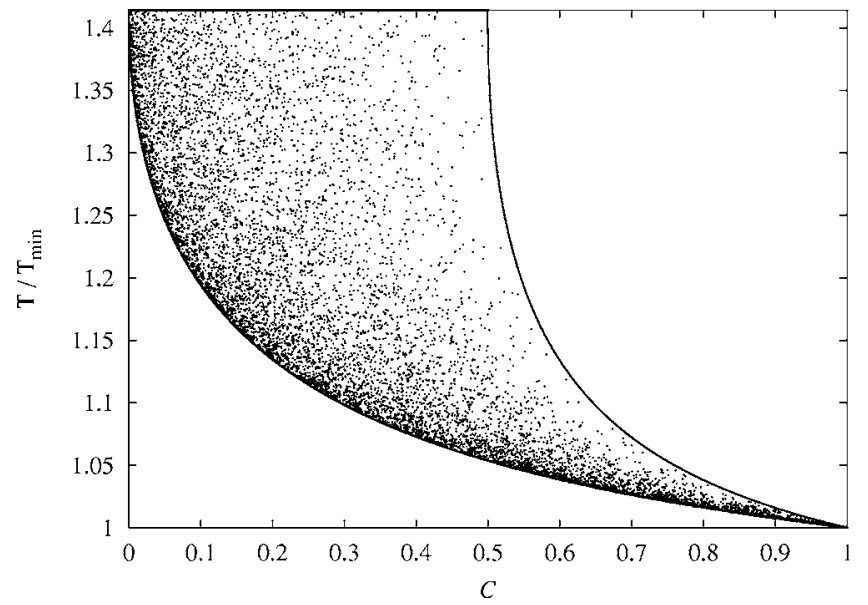

FIG. 1. Curves in the $\left(C, \tau / T_{\min }\right)$ plane corresponding, for each value of $C$, to the states of two (distinguishable) qubits with maximum and minimun $\tau / T_{\text {min }}$. The points represent randomly generated individual states that evolve to an orthogonal state. All depicted quantities are dimensionless.

mum evolution times (that is, the range of possible values for the time required to evolve to an orthogonal state) also decreases with increasing concurrence. Third, the lower bound for the evolution time to an orthogonal state is saturated by (and only by) the maximally entangled states $(C=1)$. These features provide further support to the idea that entanglement tends to speed up quantum evolution.

\section{TWO ENTANGLED INDISTINGUISHABLE PARTICLES}

Here we are going to explore the connection between entanglement and the speed of quantum evolution for systems constituted by two indistinguishable particles. In this case, the concept of entanglement exhibits some extra subtleties, as compared with the case of distinguishable subsystems. When dealing with indistinguishable particles, the correlations that arise purely from the concomitant statistics (either fermionic or bosonic) do not constitute a useful resource and, consequently, must not be regarded as contributing to the amount of entanglement of the system's state [13]. A useful formalism to describe the entanglement of systems consisting of identical particles and that takes into account the above remarks has been advanced by Eckert et al. in [13]. For two identical bosons, the system of lowest dimensionality exhibiting the phenomenon of entanglement is a pair of bosons with a two-dimensional single-particle Hilbert space. The simplest fermionic system endowed with entanglement is a system of two fermions with a three-dimensional singleparticle Hilbert space.

\section{A. Bosons}

Using the second quantization formalism, the general (pure) state of two bosons (with a two-dimensional singleparticle Hilbert space) can be written under the guise [13] of 


$$
|V\rangle=\sum_{i, j=0}^{1} v_{i j} b_{i}^{\dagger} b_{j}^{\dagger}|0\rangle,
$$

where $b_{i}^{\dagger}$ and $b_{i}$ denote bosonic creation and anhilation operators, and the coefficients $v_{i j}$ constitute the symmetric matrix

$$
\hat{V}=\left(\begin{array}{ll}
v_{00} & v_{01} \\
v_{10} & v_{11}
\end{array}\right) .
$$

That is, $v_{i j}=v_{j i}$. Normalization imposes the condition $2 \sum_{i, j=0}^{1}\left|v_{i j}\right|^{2}=1$.

The Hamiltonian associated with two noninteracting bosons is

$$
\hat{H}=\sum_{k=0}^{1} \epsilon_{k} b_{k} b_{k}^{\dagger}
$$

where $b_{0}^{\dagger}|0\rangle$ is the single-particle ground state with energy $\epsilon_{0}=0$, and $b_{1}^{\dagger}|0\rangle$ is the single-particle excited state with energy $\epsilon_{1}=\epsilon$. The state (15) evolves according to the timedependent Schroedinger equation,

$$
i \hbar \frac{d}{d t}|V(t)\rangle=\hat{H}|V(t)\rangle=\sum_{i, j=0}^{1}\left(\epsilon_{i}+\epsilon_{j}\right) v_{i j}(t) b_{i}^{\dagger} b_{j}^{\dagger}|0\rangle .
$$

The general solution of this evolution equation is given by the time-dependent coefficients,

$$
v_{i j}(t)=v_{i j}(0) \exp \left[-i \frac{\left(\epsilon_{i}+\epsilon_{j}\right)}{\hbar} t\right] .
$$

The time $\tau$ required to evolve into an orthonormal state is

$$
\langle V(0) \mid V(\tau)\rangle=2 \sum_{i, j=0}^{1}\left|v_{i j}(0)\right|^{2} \exp \left[-i \frac{\left(\epsilon_{i}+\epsilon_{j}\right)}{\hbar} \tau\right]=0 .
$$

Setting $z \equiv e^{-i(\epsilon \pi / \hbar)}=e^{-i \alpha}$, the orthogonality condition (20) can be recast as a polynomial equation in $z$ that has to admit roots of modulus equal to 1 . From this last requirement, and taking into account the symmetries in the coefficients $v_{i j}$, it follows that the coefficients can be parameterized as

$$
\begin{gathered}
\left|v_{00}\right|^{2}=\Gamma, \\
\left|v_{01}\right|^{2}=-\Gamma \cos \alpha, \\
\left|v_{11}\right|^{2}=\Gamma,
\end{gathered}
$$

with $\Gamma>0$ and $\alpha \in[\pi / 2,3 \pi / 2]$. The normalization constraints also implies that $\Gamma=1 / 4(1-\cos \alpha)$. The expectation values of the energy and its square read

$$
\begin{gathered}
E=\langle H\rangle=2 \sum_{i, j=0}^{1}\left|v_{i j}(0)\right|^{2}\left(\epsilon_{i}+\epsilon_{j}\right)=\epsilon, \\
\left\langle H^{2}\right\rangle=2 \sum_{i, j=0}^{1}\left|v_{i j}(0)\right|^{2}\left(\epsilon_{i}+\epsilon_{j}\right)^{2}=(4 \Gamma+1) \epsilon^{2},
\end{gathered}
$$

and consequently the minimum evolution time (11) is

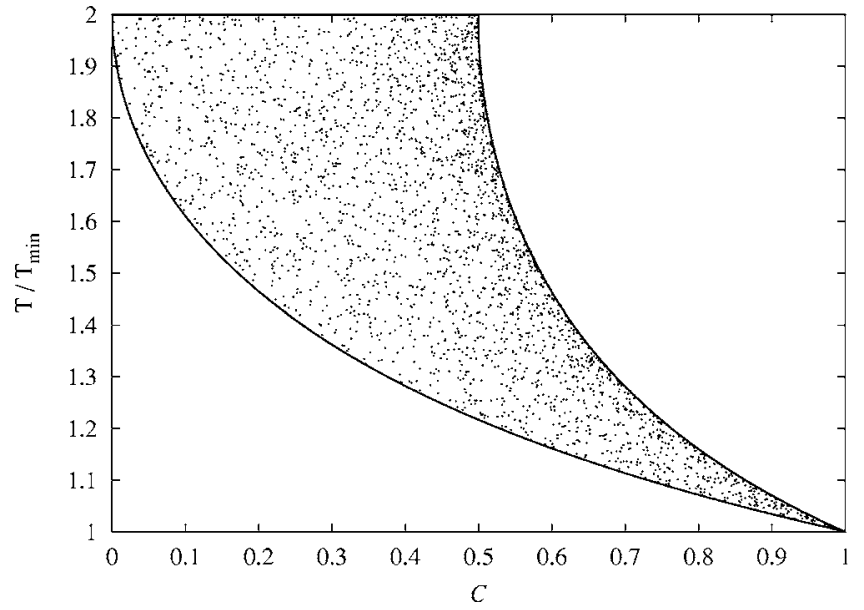

FIG. 2. Curves in the $\left(C, \tau / T_{\min }\right)$ plane corresponding, for each value of $C$, to the states of two bosons with maximum and minimun $\tau / T_{\min }$. The points represent randomly generated individual states that evolve to an orthogonal state. All depicted quantities are dimensionless.

$$
T_{\min }=\frac{\pi \hbar}{2 \epsilon}
$$

The formula for the concurrence in the two-boson case is [13]

$$
C_{B}=4\left|v_{00} v_{11}-v_{01}^{2}\right|
$$

which is clearly time independent.

For a given value of the concurrence, the minimum and maximum times for evolution to an orthogonal state can be obtained in the same way as in the case of two distinguishable qubits. The equations relating the minimum and maximum times with the concurrence are, respectively,

$$
C=\frac{1+\cos \left(\alpha_{\min }\right)}{1-\cos \left(\alpha_{\min }\right)}
$$

and

$$
C=\frac{1}{1-\cos \left(\alpha_{\max }\right)},
$$

where $\alpha_{\min , \max }=\exp \left(-i \epsilon \tau_{\min , \max } / \hbar\right)$. The curves associated with equations (25) and (26) depicting the extremum evolution times as a function of $C$, are exhibited in Fig. 2.

Comparing Fig. 2 with Fig. 1, we see that the same general trends exhibited by a system of two distinguishable qubits are also observed in the case of two identical boson.

\section{B. Fermions}

Now we are going to study a system of two identical fermions with a three-dimensional single-particle Hilbert space. In second quantization notation, the general (pure) state of such a system is 


$$
|W\rangle=\sum_{i, j=0}^{3} w_{i j} f_{i}^{\dagger} f_{j}^{\dagger}|0\rangle,
$$

where $f_{i}^{\dagger}$ and $f_{i}$ denote fermionic creation and anhilation operators, respectively, and the coefficients $w_{i j}$ constitute the antisymmetric matrix

$$
\hat{W}=\left(\begin{array}{cccc}
0 & w_{01} & w_{02} & w_{03} \\
w_{10} & 0 & w_{12} & w_{13} \\
w_{20} & w_{21} & 0 & w_{23} \\
w_{30} & w_{31} & w_{32} & 0
\end{array}\right) .
$$

That is, $w_{i j}=-w_{j i}$. Normalization imposes the condition $\sum_{i, j=0}^{3}\left|w_{i j}\right|^{2}=1 / 2$. The Hamiltonian describing two noninteracting particles is given by

$$
\hat{H}=\sum_{k=0}^{1} \epsilon_{k} f_{k} f_{k}^{\dagger},
$$

and the coefficients,

$$
w_{i j}(t)=w_{i j}(0) \exp \left[-i \frac{\left(\epsilon_{i}+\epsilon_{j}\right)}{\hbar} t\right],
$$

describe a general solution of the concomitant timedependent Schroedinger equation. Let $z \equiv e^{-i(\epsilon \tau / \hbar)}=e^{-i \alpha}$. The evolution time to an orthogonal state follows from the condition

$$
\begin{aligned}
\langle W(0) \mid W(\tau)\rangle= & 2 \sum_{i, j=0}^{3}\left|w_{i j}(0)\right|^{2} \exp \left[-i \frac{\left(\epsilon_{i}+\epsilon_{j}\right)}{\hbar} \tau\right]=4 z\left[\left|w_{01}\right|^{2}\right. \\
& +\left|w_{02}\right|^{2} z+\left(\left.w_{03}\right|^{2}+\left|w_{12}\right|^{2}\right) z^{2}+\left|w_{13}\right|^{2} z^{3} \\
& \left.+\left|w_{23}\right|^{2} z^{4}\right]=0 .
\end{aligned}
$$

The polynomial equation (31) may have either (i) fourth real roots, (ii) two real roots and two complex (complex conjugated) roots, or (iii) two pairs of complex conjugated roots. Since we are interested in solutions of the type $e^{-i(\epsilon / \hbar) \tau}$, the most general case of interest is (iii). Consequently, the two solutions of (31) corresponding to (positive) times of evolution into an orthogonal state are of the form $z_{1} \equiv e^{-i \alpha}$ and $z_{2} \equiv e^{-i \beta}$. Taking into account the antisymmetric nature of $w_{i j}$, we get the following relations:

$$
\begin{gathered}
\left|w_{01}\right|^{2}=x \\
\left|w_{02}\right|^{2}=-2 x(\cos \alpha+\cos \beta) \\
\left|w_{03}\right|^{2}+\left|w_{12}\right|^{2}=2 x(1+2 \cos \alpha \cos \beta) \\
\left|w_{13}\right|^{2}=-2 x(\cos \alpha+\cos \beta) \\
\left|w_{23}\right|^{2}=x
\end{gathered}
$$

where the value of the parameter $x$ is determined by the normalization requirement. We want to find the fastest solution to the first orthogonal state. The time $\tau$ required to reach an orthogonal state is

$$
\tau=\frac{\hbar}{\epsilon} \times \min (\alpha, \beta) .
$$

Let us consider the case $\beta=\pi$. Then, the time required to arrive to an orthogonal state is equal to $\tau=\hbar \alpha / \epsilon$ and the coefficients characterizing the quantum state are

$$
\begin{gathered}
\left|w_{01}\right|^{2}=\frac{1}{32(1-\cos \alpha)} \\
\left|w_{02}\right|^{2}=\frac{1}{16} \\
\left|w_{03}\right|^{2}+\left|w_{12}\right|^{2}=\frac{1-2 \cos \alpha}{16(1-\cos \alpha)} \\
\left|w_{13}\right|^{2}=\frac{1}{16}
\end{gathered}
$$

$$
\left|w_{23}\right|^{2}=\frac{1}{32(1-\cos \alpha)},
$$

with the obvious condition $\cos \alpha<1 / 2$ (that is, $\alpha$ $\in[\pi / 3, \pi])$. The energy and energy square expectation values read

$$
\begin{gathered}
E=\langle H\rangle=2 \sum_{i, j=0}^{3}\left|w_{i j}(0)\right|^{2}\left(\epsilon_{i}+\epsilon_{j}\right)=3 \epsilon, \\
\left\langle H^{2}\right\rangle=2 \sum_{i, j=0}^{3}\left|w_{i j}(0)\right|^{2}\left(\epsilon_{i}+\epsilon_{j}\right)^{2}=\frac{\epsilon^{2}}{2}\left[\frac{21-19 \cos \alpha}{1-\cos \alpha}\right],
\end{gathered}
$$

and, consequently, the minimum evolution time (11), after some calculation, is

$$
T_{\text {min }}=\frac{\pi \hbar}{2 \epsilon} \sqrt{\frac{2(1-\cos \alpha)}{3-\cos \alpha}} .
$$

The formula for the concurrence in the two-fermion case is [13]

$$
C_{F}=8\left|w_{01} w_{23}-w_{02} w_{13}+w_{03} w_{12}\right|,
$$

which is clearly time independent [for the Hamiltonian (29)].

One can check that the lowest value of $\tau / T_{\min }$ corresponds to $\cos \alpha=1 / 2$. That is, the state closest to saturating the lower bound for the time required to reach an orthogonal state is given by $\alpha=\pi / 3$. In this case, the fermionic concurrence reads

$$
C_{F}=\frac{\left|e^{i \varphi_{01}+i \varphi_{23}}-e^{i \varphi_{02}+i \varphi_{13}}\right|}{2},
$$

where $\phi_{i j}$ denotes the phase associated with the coefficient $w_{i j}$. Now, with an appropriate choice of the $\phi$ 's, we can make (46) either 0 or 1 . In other words, among those states that saturate the lower bound on the time to evolve to an orthogonal state, there are states of zero entanglement, as well as maximum entangled states. 


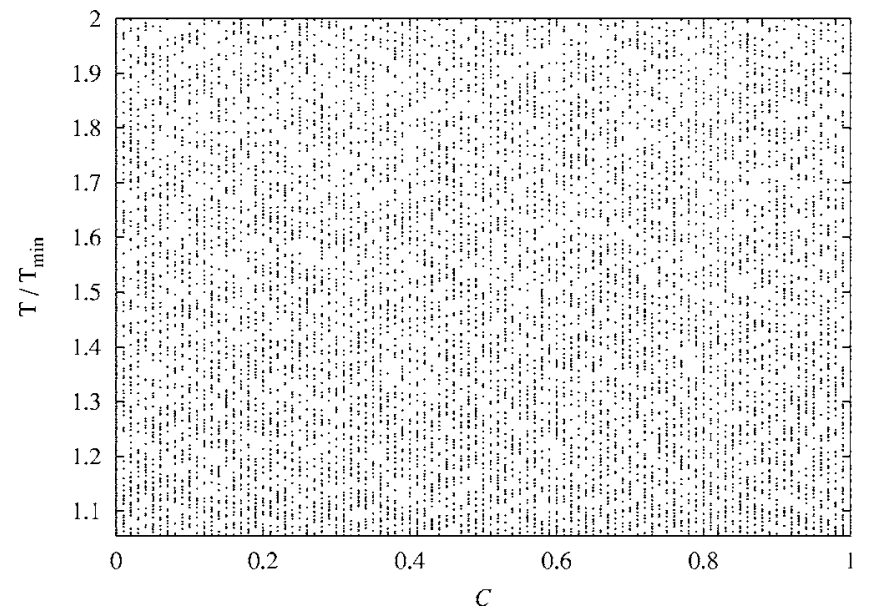

FIG. 3. Randomly generated states of two fermions that evolve to an orthogonal state. Each point corresponds to one of those states as represented in the $\left(C, \tau / T_{\min }\right)$ plane. It transpires from the figure that for each value of the concurrence $C$ the time $\tau / T_{\min }$ needed to reach an orthogonal state may adopt any value, from $\frac{1}{3} \sqrt{10}$ up to a maximum equal to 2 . All depicted quantities are dimensionless.

Figure 3 exhibits a plot in the $\left(C, \tau / T_{\min }\right)$ plane of a set of randomly generated states of two fermions that evolve to an orthogonal state. Each point represents one of those states. It transpires from the figure that for each value of the concurrence $C$, the time $\tau / T_{\min }$ needed to reach an orthogonal state may adopt any value, from $\frac{1}{3} \sqrt{10}$ up to a maximum equal to 2.

We see that, as far as the connection between entanglement and the speed of quantum evolution is concerned, the behavior of fermionic systems differs considerably from the behavior of systems consisting either of bosons or of distinguishable particles.

\section{CONCLUSIONS}

We have explored, for bipartite systems of low dimensionality, some aspects of the connection between entanglement and the speed of quantum evolution. We considered (i) two-qubits (distinguishable) systems and (ii) systems composed of two (bosonic or fermionic) identical particles with single-particle Hilbert spaces of lowest dimensionality.

Our present results corroborate that there is a clear correlation between the amount of entanglement and the speed of quantum evolution for systems of two-qubits and systems of two identical bosons. On the contrary, such a clear correlation is lacking in the case of systems of identical fermions.

\section{ACKNOWLEDGMENTS}

This work was partially supported by the MEC Grant No. BFM2002-03241 (Spain), FEDER (EU), the Government of Balearic Islands, and CONICET (Argentine Agency).
[1] V. Giovannetti, S. Lloyd, and L. Maccone, Europhys. Lett. 62, 615 (2003).

[2] V. Giovannetti, S. Lloyd, and L. Maccone, Phys. Rev. A 67, 052109 (2003).

[3] M. A. Nielsen and I. L. Chuang, Quantum Computation and Quantum Information (Cambridge University Press, Cambridge, UK, 2000).

[4] Introduction to Quantum Computation and Information, edited by H-K. Lo, S. Popescu, and T. Spiller (World Scientific, River Edge, 1998).

[5] The Physics of Quantum Information, edited by D. Bouwmeester, A. Ekert, and A. Zeilinger (Springer, Berlin, 2000).

[6] G. Alber, T. Beth, P. Horodecki, R. Horodecki, M. Röttler, H. Weinfurter, R. Werner, and A. Zeilinger, Quantum Information, Springer Tracts in Modern Physics, Vol. 173 (Springer, Berlin, 2001).

[7] Quantum Computing and Quantum Communications, edited by C. P. Williams (Springer, Berlin, 1998).

[8] C. Tsallis, S. Lloyd, and M. Baranger, Phys. Rev. A 63, 042104 (2001).

[9] J. Batle, M. Casas, A. R. Plastino, and A. Plastino, Phys. Lett. A 296, 251 (2002).

[10] J. Batle, M. Casas, A. R. Plastino, and A. Plastino, Phys. Lett. A 298, 301 (2002).

[11] J. Batle, A. R. Plastino, M. Casas, and A. Plastino, Eur. Phys. J. B 35, 391 (2003).

[12] J. Batle, M. Casas, A. Plastino, and A. R. Plastino, Phys. Rev. A 71, 024301 (2005).

[13] K. Eckert, J. Schliemann, D. Brub, and M. Lewenstein, Ann. Phys. (N.Y.) 299, 88 (2002).

[14] N. Margolus and L. B. Levitin, Physica D 120, 188 (1998).

[15] C. M. Caves and P. D. Drummond, Rev. Mod. Phys. 66, 481 (1994).

[16] S. Lloyd, Nature (London) 406, 1047 (2000). 